A N N A L E S Annales de Bretagne et des Pays de l'Ouest

\title{
Chroniques des archives du Grand-Ouest 2010-2011
}

A chronicle of the archives in the whole of Western France 2010-2011

\section{(2) OpenEdition \\ Journals}

Édition électronique

URL : http://journals.openedition.org/abpo/2603

DOI : $10.4000 / a b p o .2603$

ISBN : 978-2-7535-2782-9

ISSN : $2108-6443$

\section{Éditeur}

Presses universitaires de Rennes

\section{Édition imprimée}

Date de publication : 30 mars 2013

ISBN : 978-2-7535-2780-5

ISSN : 0399-0826

Référence électronique

"Chroniques des archives du Grand-Ouest 2010-2011", Annales de Bretagne et des Pays de l'Ouest [En ligne], 120-1 | 2013, mis en ligne le 30 mars 2013, consulté le 20 avril 2019. URL : http://

journals.openedition.org/abpo/2603 


\title{
Chronique des archives du Grand Ouest 2010-2011
}

\author{
BRETAGNE \\ Archives départementales des Côtes-d'Armor
}

Fiche signalétique

Archives départementales des Côtes-d'Armor

7 rue François-Merlet -22000 SAINT-BRIEUC

Téléphone : 0296787877 - Télécopie : 0296786729

e-mail : archives@cg22.fr

Site internet : $h t t p: / /$ archives.cotesdarmor.fr

Heures d'ouverture : du lundi au vendredi de $8 \mathrm{~h} 30$ à $17 \mathrm{~h} 30$

Pas de fermeture annuelle

Pour une première approche des fonds, consulter

Archives départementales des Côtes-d'Armor, état des fonds, 2009

(consultable sur le site internet)

\section{1) Enrichissement des collections}

\section{Archives publiques}

Parmi les versements pris en charge en 2011, il convient de signaler les ensembles suivants :

- conservation des hypothèques de Loudéac : registres indicateurs, tables alphabétiques et répertoires des formalités (1802-1952);

- archives du bureau des douanes de Saint-Brieuc (1922-2000);

- complément d'un premier versement effectué par le service historique de l'armée de terre en 2004 (partie du fonds dit " fonds de Moscou " rapatrié en France dans les années 1990) : registres matricules militaires de la subdivision de Guingamp (classes 1901-1937);

- archives du tribunal de grande instance de Saint-Brieuc (1940-2005);

- dossiers d'enseignants nés en 1920; 
- archives des anciennes écoles normales de Saint-Brieuc : comptabilité, bâtiments, intendance (1939-1983);

- archives de la chambre de commerce et d'industrie (1925-1999).

\section{Archives privées}

En dehors de pièces isolées, les deux entrées les plus notables ont été les archives professionnelles d'André Hauvespre, architecte installé à SaintBrieuc (666 projets élaborés entre 1965 et 1993), et le fonds du manoir de Trégarantec en Mellionnec (affaires familiales, gestion domaniale, activités commerciales des familles Leguen et Le Pesant, 1571-1955).

\section{Archives iconographiques et audiovisuelles}

Un bel ensemble de 9 dessins manuscrits signés de l'ingénieur Jacques Piou, représentant la maison et l'imprimerie de l'imprimeur briochin LouisJean Prud'homme (vues des façades et plans intérieurs), a permis de compléter le fonds Prud'homme. En matière d'archives orales, le partenariat avec la radio RCF Clarté a été reconduit et un exemplaire des enregistrements des émissions "Culture d'Armor " (saisons 2009-2010 et 2010-2011) a été remis aux Archives départementales.

\section{2) Instruments de recherche et publications}

\section{Instruments de recherche}

À côté des traitements réguliers (archives notariales, documents iconographiques, versements administratifs contemporains), les classements d'archives ont porté sur les ensembles suivants : archives des bureaux de l'enregistrement et des hypothèques, fonds des écoles normales, état civil de l'arrondissement de Dinan antérieur à 1940, justices de paix.

\section{3) Échos et nouvelles}

\section{Expositions}

Une exposition a été élaborée sur le thème Arts \& Archives. Les archives, sources de l'histoire des arts (septembre-décembre 2011). Elle a fait l'objet d'un dossier pédagogique abondamment illustré, consultable sur le site internet des Archives départementales.

\section{Activités pédagogiques}

3391 scolaires ont bénéficié de l'offre pédagogique des Archives (ateliers aux Archives ou dans les classes, visites du bâtiment des Archives, prêts d'expositions itinérantes sur panneaux). En 2011, le dispositif « Retour 
aux sources d'archives " a permis à 10 classes du primaire et du secondaire d'élaborer des travaux de recherche en lien avec l'histoire locale.

\section{Fréquentation de la salle de lecture}

La fréquentation de la salle de lecture accuse une baisse régulière depuis 2006 (début de la mise en ligne de l'état civil numérisé). Ainsi, 1489 lecteurs se sont inscrits ou réinscrits en 2011, chiffre en légère baisse rapport à 2010 (-6,7\%), tandis que le nombre de communications est resté stable (19782 documents consultés en 2011, 19384 en 2010). Le nombre de séances de travail, équivalent à des entrées, s'est élevé à 3155 , en baisse de 23,3 \% par rapport à 2010 (4112).

\section{Site Internet}

En complément de la mise en ligne des vues numérisées de l'état civil et des listes nominatives de recensement de la population, le partenariat avec le Centre généalogique des Côtes-d'Armor a permis d'enrichir le contenu de la base de données Généarmor (plus de 6 millions d'actes analysés à partir des registres paroissiaux et d'état civil). La numérisation du recensement de la population de 1906 a donné lieu à la mise en ligne d'une base de données nominatives relatives à près de 600000 individus (Censo-Net), interrogeables sur le site de l'association (http://www.genealogie22.org). La conséquence de ces mises en ligne massives de données numérisées et textuelles continue à se traduire par une fréquentation très importante du site internet des Archives départementales (1,3 million de visites et près de 127 millions de pages vues en 2011).

\section{Numérisation}

Le programme de numérisation a été poursuivi en 2011 et a concerné, outre les compléments de fonds déjà numérisés (registres d'état civil, listes nominatives de recensement de population), la photothèque du fonds d'archives de l'homme politique Charles Josselin, le fonds photographique Hyacinthe Bolloc'h (portraits, des scènes familiales, vues d'expositions universelles et un ensemble de 78 clichés concernant la première guerre mondiale pris probablement par un opérateur photographe attaché à l'Armée) et les registres matricules militaires (1867-1909). 


\section{Archives départementales du Finistère}

\begin{tabular}{|c|} 
Fiche signalétique \\
Archives départementales du Finistère \\
Dépôt de Quimper \\
allée Henri-Bourde-de-La-Rogerie, 29000 QUIMPER \\
Dépôt annexe de Brest \\
1 rue Jean-Fouchet -29200 BREST \\
Téléphone : 0298959191 - Télécopie : 0298953169 \\
e-mail : archives.departementales@cg29.fr \\
Heures d'ouverture : site de Quimper, du lundi au vendredi de $8 \mathrm{~h} 30$ à $17 \mathrm{~h} 00$ \\
(interruption des levées de $12 \mathrm{~h} 00$ à $13 \mathrm{~h} 00$ et à partir de $16 \mathrm{~h} 00)$. Fermeture le \\
dernier vendredi du mois. \\
site de Brest, du lundi au vendredi de $8 \mathrm{~h} 30$ à $12 \mathrm{~h} 00$ et de $13 \mathrm{~h} 00$ à $17 \mathrm{~h} 00$
\end{tabular}

\section{1) Enrichissement des collections}

\section{Archives publiques}

Parmi les versements pris en charge en 2011, on peut noter les ensembles suivants :

- conservation des hypothèques, circonscription de Brest, premier bureau : registres (1877-1956);

- conseil général du Finistère, direction de l'Assemblée et du partage de l'information : enregistrement sur cassettes audio des séances de l'assemblée délibérante (1981-1988);

- registres de l'état civil des tribunaux de grande instance de Brest (pour la période 1874-1910) et de Quimper (pour la période 1863-1912).

Concernant les dépôts d'archives communales, les Archives départementales du Finistère ont enregistré les dépôts des communes suivante : Arzano, Botmeur, Briec, Châteauneuf-du-Faou, Elliant, Ergué-Gabéric, Esquibien, Leuhan, Melgven, Plouhinec, Plobannalec-Lesconil, Pluguffan, Port-Launay, Saint-Yvi.

On soulignera un effort important en direction du patrimoine maritime contemporain notamment.

\section{Archives privées}

Parmi les dépôts faits en 2011, on peut signaler tout particulièrement :

- quelques pièces de la correspondance de l'abbé Madec sur le Bleun Brug (1926-1928);

- deux fonds d'association de protection de la nature et de l'environnement : le fonds de l'Union régionale bretonne pour l'environnement (URBE), et le fonds du Comité de défense des sites de Moëlan-sur-Mer; 
- le fonds déposé d'architecte, le fonds Erwan Le Berre, architecte à Quimper;

- le fonds Guy Maillot (archives d'un militant CGT);

- le fonds de la section brestoise de l'Association des déportés et internés du Finistère (journaux d'activité, comptabilité, administration, dossiers individuels).

\section{Archives iconographiques et audiovisuelles}

Les archives iconographiques s'enrichissent régulièrement de cartes postales, de négatifs photographiques, de dessins ou d'estampes. Pour 2011, au total, les Archives du Finistère ont acquis 121 cartes postales, 37 négatifs photographiques, 20 photographies et 4 estampes.

Deux dons de documents audiovisuels sont à signaler, ils constituent les premiers dons de ce type aux Archives du Finistère : un film super-8 (1974) en couleur ayant pour thème des vacances dans le Finistère (pointe du Raz, Quimper, Brest, festival du Ménez-Hom), et un ensemble de films noir-et-blanc Pathé Baby (années 1920-1930) sur Gouézec, Pleyben et sa région, et représentant des pardons, fêtes religieuses et festivals de gymnastique.

\section{2) Instruments de recherche, publications, bases de données}

Les Archives départementales du Finistère n’ont produit aucune publication en 2011.

Concernant les instruments de recherche, un gros effort a été fourni pour rétroconvertir au format XML/EAD de nombreux instruments de recherche (inventaires et états des fonds), afin de préparer l'ouverture du site Internet du service. Parmi ces inventaires, on citera des bases thématiques sur les affiches de la guerre 1914-1918, les répertoires des fonds phares et balises, sur la langue bretonne, le Canal de Nantes à Brest, les mines de plomb argentifère de Poullaouen et Huelgoat et le cadastre napoléonien, certains avec des liens intégrés vers les documents numérisés.

L'année 2011 a été marquée par les travaux liés au site Internet des Archives du Finistère, dont l'ouverture a été prévue en 2012. Les travaux ont porté sur la préparation des pages et la réflexion sur la structure du site, ainsi qu'un gros travail de rétroconversion au format électronique des instruments de recherche papier, et de numérisation pour les inventaires trop complexes ou mal structurés.

\section{3) Échos et nouvelles}

\section{Activités pédagogique}

En raison de l'absence de professeur du service éducatif, seuls 75 élèves ont pu bénéficier d'un accueil dans le service (52 de classes élémentaires, 
23 collégiens). 127 personnes d'autres publics ont également bénéficié de visites et de présentation du service (étudiants et membres d'associations).

\section{Partenariat}

Un partenariat doit particulièrement être évoqué pour 2011 : les Archives départementales du Finistère ont collaboré avec l'Association pour l'histoire de la protection de la nature et de l'environnement (AHPNE) en apportant leur aide technique à une vacataire recrutée par l'association. Ce partenariat a débouché sur la création d'un guide des sources qui a été élaboré sous forme papier et électronique.

\section{Fréquentation de la salle de lecture}

Les salles de lecture de Quimper et Brest ont enregistré 7368 séances de travail, pour 29469 documents communiqués.

\section{Numérisatiobn}

Parmi les travaux de numérisation, on pourra citer la numérisation des enregistrements des séances de l'assemblée délibérante du conseil général du Finistère, ainsi qu'une partie de la presse politique et d'information générale (1880-1944); ces deux opérations se poursuivent en 2012.

\section{Archives départementales d'Ille-et-Vilaine}

\begin{tabular}{|c|} 
Fiche signalétique \\
Archives départementales d'Ille-et-Vilaine \\
Adresse postale : Direction des Archives et du Patrimoine \\
1, rue de la préfecture, CS 2418 - 35041 RENNES Cedex 1 \\
Adresse topographique : 1 rue Jacques-Léonard - 35000 RENNES \\
Téléphone : 029902 40 00 - Télécopie : 0299024001 \\
E-mail : archives@cg35.fr \\
Site internet : www.archives35.fr \\
Heures d'ouverture : du lundi au vendredi de 8h30 à 17h30 \\
(sauf le 1 $1^{\text {er }}$ lundi matin de chaque mois) \\
(Fermeture annuelle la première quinzaine d'août) \\
Pour une première approche des fonds, consulter : \\
Guide des archives d'Ille-et-Vilaine, tome 1, volume 1, 1994 \\
(fonds antérieur à la Révolution)
\end{tabular}




\section{1) Enrichissement des collections}

\section{Archives publiques}

Les archives contemporaines ont connu une baisse des entrées : $585 \mathrm{ml}$ en comparaison des $1075 \mathrm{ml}$ de 2009 et $1500 \mathrm{ml}$ de 2010, ce qui permet de résorber progressivement les entrées massives qui ont suivi la réforme de l'administration territoriale de l'Etat (RéAte).

Les visas d'élimination réglementaire des archives arrivées au terme de leur durée d'utilité administrative et dénuées d'intérêt historique ont atteint les 10600 mètres linéaires.

Le groupe de travail réunissant, depuis 2010, les archivistes chargés des archives contemporaines dans les quatre services d'Archives départementales a poursuivi ses travaux notamment en matière de communicabilité des fonds.

Pour l'année 2011, peuvent être signalés :

- Les entrées témoignant des activités du conseil général (40 ml), dont les plus importantes concernent le secteur social comprenant les échantillons des dossiers d'aide sociale en faveur des personnes âgées et personnes handicapées de 1957 à 2011, différents ensembles en provenance des CDAS et du service de la protection de l'enfance - dont on peut retenir le suivi des établissements et des services de l'enfance de 1950 à 2001 ; sont également bien représentés le service foncier des infrastructures - comprenant le répertoire général des actes administratifs du département de 1986-2005, et les services culturels - avec les dossiers de subventions en matière de conservation des antiquités et objets d'art de 1982 à 1996 et les subventions aux bâtiments communaux de 1989 à 2003.

- Les versements dans le secteur sanitaire et social : au niveau régional figurent les documents relatifs aux commissions exécutives de l'Agence régionale de santé, les "reliquats " de la direction régionale de l'action sanitaire et sociale (DRASS) de Bretagne qui comprennent notamment des enquêtes ( $7 \mathrm{ml}$ ) ou des fonds de dossiers sur les établissements remontant aux années 1960. $38 \mathrm{ml}$ concernent les différents organismes de sécurité sociale de 1933 à 2006 (notamment les fonds évacués du centre d'archivage du Mans qui a fermé ses portes).

Au niveau départemental, $38 \mathrm{ml}$ de documents proviennent des services de la direction départementale de l'action sanitaire et sociale (DDASS) : ils touchent aux professions de santé, aux soins (notamment la santé mentale de 1952 à 2006), à l'environnement (notamment les comptes rendus du CODERST de 1958 à 2004).

- Dans le secteur agricole, on peut remarquer les versements de la direction régionale de l'agriculture concernant tant les recensements et enquêtes que l'économie par le biais des contrats de plans de 1966 à 1985 ou encore des dossiers de marchés des industries agro-alimentaires (abattoirs) de 1960 à 2000. 
- Le premier versement de la direction interrégionale des Anciens combattants : il concerne le fichier des pensionnés (1875-2008), un échantillon des dossiers de pension, les commissions de réforme.

- Le versement de l'INSEE relatif à l'enquête du recensement de 2005.

- Les documents en provenance des diverses administrations judiciaires : le tribunal administratif, la cour d'appel (39 $\mathrm{ml}$ de dossiers de procédure) et le tribunal de grande instance de Saint-Malo (73 ml de procédures correctionnelles de 1981 à 2000).

- Les documents des services de l'équipement relatifs aux documents d'urbanisme relevant de l'autorité de l'État provenant du service de SaintMalo (1984 à 2001).

- les $117 \mathrm{ml}$ de documents des services fiscaux parmi lesquels : les fonds de l'enregistrement (déclarations de successions, fiches de décès, bordereaux des actes...) des bureaux de Redon (1937-2007) et de Saint-Malo nord (1942-2004).

- Plus de $90 \mathrm{ml}$ en provenance de la préfecture : on relève les 33 premiers mètres linéaires de dossiers d'installations classées du secteur agricole (1931-2011), le premier registre des associations (1934-1954) accompagnés de près de $10 \mathrm{ml}$ de dossiers d'associations ou syndicats dissous, les dossiers et procès-verbaux de la commission départementale des sites (1979-2006), les dossiers et statistiques de la commission départementale d'équipement commercial (2003-2008), les procès-verbaux et listes d'émargement des élection régionales de 2010 et cantonales de 2011.

- Le premier versement touchant à l'administration et les activités de formation du CREPS de Dinard (1946-2007).

- Le versement du centre d'information sur l'énergie et l'environnement (CIELE) relatif aux activités de l'association et à ses actions pédagogiques de 1984 à 2011.

- Plusieurs études notariales, qui n'avaient pas encore versé (SaintMéen-le-Grand, Servon-sur-Vilaine, minutes des XVIII et XIX ${ }^{\mathrm{e}}$ siècles), ou qui ont complété leurs versements (4 études, période 1900-1936, dont l'importante étude de Bain-de-Bretagne, près de $90 \mathrm{ml}$ pour la seule période 19001936).

\section{Archives privées}

Parmi les fonds privés entrés en 2011, on peut notamment citer un supplément du fonds des syndicats médicaux d'Ille-et-Vilaine, le fonds Morice (une partie des archives du tribunal militaire allemand de Rennes, 19401944), le fonds du militant écologiste Dominique Bernard (224 J), les fonds des érudits Jean Le Bras (Montreuil-sur-Ille, 214 J), Léon Pérouas (Vernsur-Seiche, 216 J), Marcel Donet (Saint-Médard-sur-Ille, 220 J), de l'ancien conseiller général Martial Gabillard (219 J), le don par Bertrand Frélaut de divers périodiques du Mouvement breton de 1946 à 1972 (218 J). Il faut enfin signaler la signature de la convention de dépôt des archives de l'UDCGT d'Ille-et-Vilaine. 


\section{Fonds iconographiques}

Trois entrées à signaler pour 2011 :

- achat de la collection de cartes marines éditée par le service hydrographique et océanographique de la Marine en 2011 concernant la Bretagne (fonds coté 7 Fi 7/45);

- don de la production graphique du graphiste Mathieu Desailly. Ce don comprend à la fois les affiches produites, les supports de communication du type plaquettes, marque-pages, CD... et des archives permettant d'appréhender le processus de création (ektachromes, fichiers électroniques). Le graphiste étant toujours en activité, le fonds, coté $44 \mathrm{Fi}$, sera complété chaque année par sa production annuelle;

- don du fonds Maurice Jannin : collection de 26 affiches illustrées relatives à la première guerre mondiale (fonds coté $42 \mathrm{Fi}$ ).

\section{Bibliothèque}

La bibliothèque a continué de s'enrichir (301 ouvrages achetés en 2011) et reçoit 88 revues scientifiques.

\section{2) Instruments de recherche}

\section{Instruments de recherche}

Pour les archives postérieures à 1940, outre les bordereaux de versements qui accompagnent tous les fonds entrés, les traitements de 2011 ont permis la réalisation de 107 répertoires et de référencer ainsi plus de $750 \mathrm{ml}$ de documents. Les priorités de traitement portent toujours sur des archives judiciaires, les fonds de l'enregistrement et de la préfecture.

En ce qui concerne les archives modernes (1800-1940), le traitement des archives judiciaires s'est poursuivi par le fonds du tribunal de première instance de Rennes (3 U 4).

En matière d'archives privées, la plupart des fonds entrés ont fait l'objet d'inventaires peu de temps après leur entrée.

Pour les archives communales, citons les classements des archives des communes de Bains-sur-Oust, La Bosse-de-Bretagne, Landavran, RozLandrieux, Saint-Malon-sur-Mel. Le classement des archives de Dol-deBretagne, fait en 1984, a été entièrement repris et complété en vue d'un transfert à la médiathèque de la ville.

\section{Bases de données}

De nombreux instruments de recherche, notamment en archives contemporaines et modernes (citons pour ces dernières plusieurs bureaux de l'administration de l'enregistrement et plusieurs études notariales), ont été mis en ligne et sont accessibles sur le site Internet (cf. infra) à partir de la rubrique " archives en ligne ". 


\section{3) Échos et nouvelles}

\section{Expositions}

L'exposition consacrée à l'œuvre de l'architecte Arthur Regnault, architecte du XIX ${ }^{\mathrm{e}}$ siècle qui a marqué de son empreinte le patrimoine religieux du département, a été accompagnée par un cycle de conférences et une publication en coédition avec les Presses universitaires de Rennes : Arthur Regnault, architecte (1839-1932), La quintessence de l'art sacré, sous la direction de Jean-Yves Andrieux. Cette exposition a attiré près de 10000 visiteurs en 2011 dans le bâtiment des Archives et sur les territoires, dans sa version itinérante (communes, propriété départementale du sémaphore de la pointe du Grouin).

Une exposition a été proposée aux habitants de Saint-Senoux à partir du classement des archives de la commune à l'issue de leur classement et a donné lieu au numéro 3 de la série " Archives et histoire ".

Les rendez-vous "Bande dessinée et Histoire " s'installent dans la programmation avec "Paroles d'étoiles, mémoires d'enfants cachés " du scénariste Serge Le Tendre, exposition qui a attiré, avec le rendez-vous précédent consacré aux pirates et corsaires, plus de 2000 visiteurs, dont 1535 scolaires.

Il convient enfin de signaler la fréquentation importante lors des Journées européennes du patrimoine 2011 (plus de 900 personnes) et le succès constant que connaissent les visites commentées du bâtiment des Archives départementales chaque mois.

\section{Service éducatif}

Le service éducatif des Archives départementales a reçu 538 élèves. Trois professeurs (arts plastiques, histoire, français) y ont animé des ateliers sur l'affaire Dreyfus, le camp de nomades de Rennes durant la seconde guerre mondiale, la propagande sous le régime de Vichy et le Centre dramatique de l'Ouest. L'atelier de calligraphie a poursuivi son activité avec 462 participants.

\section{Fréquentation de la salle de lecture}

En 2011, 1800 lecteurs ont consulté plus de 22000 documents originaux.

\section{Site Internet}

En 2011, le site internet des Archives départementales d'Ille-et-Vilaine [www.archives35.fr] a été fréquenté par 173570 lecteurs uniques qui ont consulté 1834161 pages lors de 778255 visites. Cette année a vu également une notable amélioration du système de visualisation qui présentait auparavant des défaillances. Les derniers lots d'images des registres d'état civil ont été intégrés. Cette évolution a entraîné le report de la publication des 
fonds figurés et des vues des recensements de la population, entre 1836 et 1911.

Une exposition virtuelle dynamique a été créée et mise en ligne à partir de l'exposition Verso, mettant en scène les travaux du graphiste rennais Mathieu Desailly.

Quatre conférences autour de l'exposition Arthur Regnault sont également visibles sur le site. Toujours dans le cadre de cette exposition, les Archives d'Ille-et-Vilaine proposent également une application à télécharger pour Smartphone afin de découvrir trois circuits de visite géolocalisés dans l'ensemble du département présentant des églises remarquables d'Arthur Regnault.

\section{Numérisation}

Les deux principales opérations de numérisation à signaler en 2011 sont les 5546 diapositives du fonds Meirion-Jones (40 Fi, photographies de l'habitat vernaculaire en Bretagne) et 65025 vues de registres matricules militaires (sous-série 1 R) pour les classes de 1878 à 1900.

\section{Archives départementales du Morbihan}

\section{Fiche signalétique}

Archives départementales du Morbihan

80 rue des Vénètes - 56010 VANNES cedex Téléphone : 0297463252 - Télécopie : 0297464876

e-mail : archives@cg56.fr

Site internet : $w w w$.archives.morbihan.fr

Heures d'ouverture : du lundi au vendredi $9 \mathrm{~h} 00-17 \mathrm{~h} 30$

Fermeture mensuelle le $2^{\mathrm{e}}$ mercredi matin de chaque mois

Fermeture annuelle du 15 au 31 décembre

\section{1) Enrichissement des collections}

En 2011, les collections des Archives départementales du Morbihan se sont enrichies de $568 \mathrm{ml}$ de documents d'archives.

Parmi les versements pris en charge en 2011, on peut noter les ensembles suivants :

- les archives de l'Inspection d'académie (1858-1961);

- les dossiers individuels des prévenus du commissariat de Lorient (1869-1952); 
- les archives de la direction départementale de l'Équipement maritime (1940-1989);

- une campagne photographique des côtes morbihannaises [années 1960-1970];

- les archives des phares et balises (1940-1990);

- les dossiers de personnel de la Poste (1825-1940);

- les archives du tribunal de commerce de Vannes (1919-1998);

- les procès-verbaux et dossiers de la commission départementale des sites (1974-2009);

- les archives du tribunal maritime de commerce de Lorient (1940-2008).

\section{Archives privées}

En 2011, la bibliothèque du château de Rochefort-en-Terre (XvI ${ }^{\mathrm{e}}$ $\mathrm{xx}^{\mathrm{e}}$ siècles), $40 \mathrm{ml}$, appartenant au peintre américain Klotz a intégré les collections des Archives du Morbihan.

\section{2) Instruments de recherche et publications}

En 2011, $830 \mathrm{ml}$ ont été classés et 116 instruments de recherches ont été produits. A été ainsi classé l'ensemble des archives des tribunaux d'instance et des tribunaux de commerce couvrant la période 1930-2003. En outre, sont désormais devenus accessibles, entre autres documents, le fonds de l'école nationale de police (1972-2010) ainsi que celui du centre d'éducation spécialisée de Belle-l̂le (1948-1998). Le catalogue de la bibliothèque du service a été mis en ligne soit quelque 64000 notices décrivant ainsi l'intégralité de la collection.

En parallèle, les Archives du Morbihan ont publié un catalogue d'exposition Récits de voyage : le Morbihan, une destination exotique présentant les impressions d'auteurs célèbres ou anonymes lors de leurs séjours dans le Morbihan durant trois siècles.

\section{3) Échos et nouvelles}

\section{Expositions}

Les Archives départementales ont proposé du 15 février au 14 décembre 2011 une exposition intitulée Récits de voyage : le Morbihan, une destination exotique. S'appuyant sur les nombreux récits de voyages conservés par la bibliothèque des Archives départementales, cette exposition avait pour objectif de présenter le regard porté sur le département par des écrivains voyageurs, célèbres ou anonymes, du XvII ${ }^{\mathrm{e}}$ au début du XXe ${ }^{\mathrm{e}}$ siècle. 2533 personnes se sont déplacées pour la voir, dont 819 scolaires. Des visites guidées ont été organisées au profit des maisons de retraite et associations du département, elles ont profité à 216 personnes. 
Parallèlement, les Archives mettent gratuitement à disposition des organismes culturels et associatifs les expositions préalablement présentées dans leur hall. En 2011, les Archives du Morbihan ont ainsi réalisé 55 prêts d'expositions. Près de 12000 personnes les ont visitées.

\section{Activités pédagogiques}

Le service éducatif sensibilise un grand nombre d'élèves morbihannais au travers de multiples activités (ateliers, travaux sur documents, visites...). Ainsi, près de 6000 élèves en 2011 ont bénéficié des actions du service éducatif. Cependant, l'Inspection académique du Morbihan a supprimé le poste d'enseignant-relais des écoles publiques à la rentrée 2011 assombrissant du même coup l'avenir du service éducatif.

\section{Fréquentation de la salle de lecture}

Le nombre de lecteurs fréquentant la salle de lecture a poursuivi sa baisse en 2011, dans des proportions moindres que les années précédentes. 1755 lecteurs se sont inscrits ou réinscrits en 2011 pour consulter 20000 documents. En termes de séances de travail, équivalentes à des entrées, cela représente 4900 lecteurs. La fréquentation en baisse de la salle de lecture est plus que compensée par l'affluence que connaît le site Internet des Archives départementales : plus de 214000 visiteurs et plus de 643000 visites. Les Archives du Morbihan ont d'ailleurs complété l'offre documentaire mise à disposition des internautes en mettant en ligne en septembre 2011 plus de 238000 pages appartenant aux registres de l'état civil du dernier tiers du XIX ${ }^{\mathrm{e}}$ siècle.

\section{Numérisation}

Les Archives du Morbihan ont poursuivi la numérisation des registres matricules de recrutement militaire en reproduisant les registres des classes 1873-1892. Parallèlement, les listes de recensement de population (1805-1911) ont été numérisées. 


\section{PAYS DE LA LOIRE}

\section{Archives départementales de Loire-Atlantique}

\begin{tabular}{|c|}
\hline Fiche signalétique \\
Archives départementales de Loire-Atlantique \\
6, rue de Bouillé, BP 23505 - 44035 NANTES Cedex 1 \\
Téléphone : 0251 729320 - Télécopie : 0240202691 \\
E-mail : archives@cg44.fr \\
Internet : www.loire-atlantique.fr rubriques Au quotidien, Culture, \\
Archives départementales \\
Heures d'ouverture : lundi, mercredi, jeudi, vendredi $9 \mathrm{~h} 00-17 \mathrm{~h} 00$, mardi \\
13h30-19h00 (17h00 en période de vacances scolaires) \\
Fermeture annuelle la première quinzaine d'août et du 25 décembre au $1^{\mathrm{er}}$ janvier inclus \\
Pour une première approche des fonds, consulter : \\
Guide des archives, Nantes, 1962-1964, 2 vol. \\
\hline
\end{tabular}

\section{1) Enrichissement des collections}

\section{Archives publiques}

Parmi les versements pris en charge en 2011, on peut noter les ensembles suivants :

- base aérienne et aérodrome de Montoir (1923-2009);

- hospitalisations psychiatriques (1923-1998);

- procès-verbaux du conseil d'administration (1918-1979) de la succursale de la Banque de France de Saint-Nazaire;

- tables des rôles d'équipage quartiers de Saint-Nazaire (1882-1904) et de Nantes (1882-1936), rôles d'équipage des quartiers de Nantes, SaintNazaire et Le Croisic (1929-1940), et matricules des gens de mer des quartiers de Nantes et de Saint-Nazaire (1882-1950);

- travaux de routes départementales (1929-1982);

- centre de formation de la police nationale (1986-2009);

- maison départementale de Mindi (1862-2000);

- lycée professionnel Livet (1890-1970);

- procès-verbaux du conseil d'administration de l'université de Nantes (1917-2006);

- divers organismes de sécurité sociale, d'allocations familiales et de retraite (1950-2000);

- inspections des entreprises de plus de 50 salariés (1983-2008).

\section{Archives privées}

Deux ensembles d'origine privée méritent d'être signalés au titre de l'année 2011 : les archives de la seigneurie de Lauvergnac en Guérande $\left(\mathrm{XVI}^{\mathrm{e}}-\mathrm{XVIII}{ }^{\mathrm{e}}\right.$ siècles), et des documents concernant une exploitation sucrière 
à Saint-Domingue, propriété d'une famille nantaise du XVIIle siècle (correspondance principalement avec le régisseur, comptes particulièrement intéressants pour les informations qu'ils apportent sur les produits échangés, la population esclave...). Par ailleurs, différents contacts ont été établis, qui devraient entraîner, en 2012, l'entrée de fonds intéressants : papiers du résistant Émile Letertre, archives de Jean-Louis Boistel (tailleur de pierre), fonds de l'entreprise Mottais de Saint-Nicolas de Redon (menuiserie navale), correspondance d'Ange Guépin.

\section{Archives iconographiques et audiovisuelles}

Plusieurs ensembles iconographiques dignes d'intérêt sont venus enrichir les fonds et collections : 1800 plaques de verre stéréoscopiques de la fin $\mathrm{XIX}^{\mathrm{e}}$-début $\mathrm{XX}$ e siècle témoignant de scènes de vie familiale et des voyages du marchand de bois nantais Hailaust; 60 tirages des bombardements de SaintNazaire de 1943; 600 photos du fonds de tôlerie Thiriet \& Cie; et une dizaine de vues de manifestations regroupant les jeunesses patriotiques après la dissolution des ligues (1928-1938).

La convention avec la Cinémathèque de Bretagne a permis le dépôt de films faisant partie des fonds et collections des Archives départementales (10 films réalisés par les anciens services rattachés aujourd'hui à la direction régionale de l'environnement, de l'aménagement et du logement, archives audio-visuelles du fonds Liard sur le théâtre, cassettes du fonds de l'association Entreprise et Patrimoine Industriel). Par ailleurs, ont été collectés un important fonds de 95 films sonorisés réalisés par Justin Leduc agriculteur à Saint-Père-en-Retz (vie à la campagne, laiterie, paludier, ostréiculture...), des images de la caisse EDF provenant du Centre d'histoire du travail de Nantes et un film sur une kermesse à Touvois en 1964.

\section{2) Instruments de recherche et publications}

En dehors des traitements récurrents (minutiers de notaires, documents figurés, versements courants d'archives administratives, fonds privés), les classements d'archives ont porté sur les volumineux versements des archives des bureaux de l'Enregistrement des années 1960, tandis que le fonds de l'entreprise de conserverie Saupiquet a fait l'objet d'un récolement détaillé.

Le catalogue informatisé de la bibliothèque des Archives comprend désormais celui de la bibliothèque administrative, suite à l'intégration du son fichier manuel. Le fichier matières de la bibliothèque historique antérieur à 1992 a fait l'objet d'une conversion rétrospective, qui permettra à terme d'intégrer près de 25000 notices dans le catalogue informatisé. 


\section{3) Échos et nouvelles}

\section{Expositions}

Deux expositions ont été présentées, Nantes - cour d'assises - 1931. Le procès des insurgés de Cayenne, du 8 février au 26 juin 2011, et Armateurs d'arts : les Dobrée, du 14 septembre au 18 décembre 2011. Elles ont fait chacune l'objet d'un numéro spécial du journal d'information Liens d'archives, faisant office de catalogue.

\section{Activités pédagogiques}

5563 scolaires ont bénéficié de l'offre pédagogique des Archives représentant, originaires de quelque 70 établissements d'enseignement. 357 élèves de 12 établissements différents ont bénéficié d'ateliers délocalisés dans les classes elles-mêmes, tandis qu'il a été permis à 3510 autres élèves de profiter de prêts d'expositions itinérantes sur panneaux. À signaler un nouvel atelier consacré à la reliure.

\section{Fréquentation de la salle de lecture}

2291 lecteurs se sont inscrits ou réinscrits en 2011, chiffre stable par rapport à 2010, tandis que le nombre de communications a sensiblement augmenté passant de 19720 documents consultés (hors consultations des fonds numérisés en salle multimédia) à 23000, soit une progression de $15 \%$. Le nombre de séances de travail, équivalent à des entrées, s'est élevé à 9493, en très légère hausse par rapport à 2010.

\section{Numérisation}

Les opérations de numérisation ont porté essentiellement sur des compléments de fonds déjà numérisés (plans cadastraux, registres d'état civil, délibérations communales, répertoires chronologiques des notaires).

Le projet d'océrisation de la presse d'information locale est entré dans une phase opérationnelle, avec le traitement de L'Écho de la Loire entre 1920 et 1932. Il offrira aux chercheurs, en salle de lecture et sur internet, un produit innovant de consultation de la presse, avec la possibilité de faire des recherches directement sur le texte des articles. 


\section{Archives départementales de Maine-et-Loire}

\begin{tabular}{|c|}
\hline Fiche signalétique \\
Archives départementales de Maine-et-Loire \\
106 rue de Frémur, BP 80744 - 49007 ANGERs cedex 1 \\
Téléphone : 0241808000 - Télécopie : 0241685863 \\
e-mail : archives49@cg49.fr \\
Site internet : www.archives49.fr \\
Heures d'ouverture : lundi : $13 \mathrm{~h} 30-18 \mathrm{~h} 00$, mardi-vendredi : $9 \mathrm{~h} 00-18 \mathrm{~h} 00$ \\
arrêt des communications entre $12 \mathrm{~h} 00$ et $13 \mathrm{~h} 30$ et après $17 \mathrm{~h} 00$ \\
(fermeture annuelle la première quinzaine d'août)
\end{tabular}

Les Archives départementales conservent près de 40 kilomètres de documents d'archives, couvrant une large période chronologique, du $\mathrm{IX}^{\mathrm{e}}$ siècle à nos jours. Les fonds médiévaux ecclésiastiques (abbayes de Fontevraud, de Saint-Florent...) sont particulièrement riches. Les fonds privés sont également très divers (familles, entreprises, associations...). La bibliothèque historique comprend plus de 13000 volumes et se présente comme un utile complément à la recherche.

\section{1) Enrichissement des collections}

840 mètres linéaires de documents ont été collectés en 2011, ainsi que près de 300 heures d'enregistrements sonores.

\section{Archives publiques}

Parmi les nombreux versements entrés en 2011, on peut signaler les ensembles suivants :

- tribunal de commerce d'Angers : dossiers d'immatriculations, dépôts des actes de sociétés, agents commerciaux (1954-2007);

- inspection académique : bourses, examens et concours, inspection, enseignants, élèves (1940-2002);

- institut universitaire de formation des maître d'Angers : scolarité, personnel, ensemble de planches pédagogiques du laboratoire (XIX ${ }^{\mathrm{e}}$ siècle-2000);

- organismes sociaux : CAF, CPAM et URSSAF d'Angers et de Cholet (1930-3004).

\section{Archives privées}

À signaler parmi les dons et dépôts, les fonds suivants qui apportent un éclairage sur l'histoire contemporaine du département :

- $334 \mathrm{~J}$ : parti communiste français, fédération du Maine-et-Loire, entré par dépôt ( $\mathrm{Xx}^{\mathrm{e}}$ siècle); 
- $335 \mathrm{~J}$ : fédération des centres sociaux et socio-culturels de Maine-etLoire, entré par dépôt (1972-2011).

Sont également entrés par dépôts de nombreux dossiers clients provenant de l'étude Magdeleine à Angers ( $\mathrm{XvI}^{\mathrm{e}}-\mathrm{xX}^{\mathrm{e}}$ siècle).

Parmi les achats, a notamment été acquis le censif de Mûrs (1377-1392).

\section{Archives iconographiques et audiovisuelles}

- Documents iconographiques : plans, cartes postales et photographies, notamment aériennes, relatives aux communes du département; collection de faire-part de décès de personnalités angevines (1922-1970); monuments : vues du château de Montsoreau et d'autres monuments angevins réalisés par le marquis de Geoffre, dessins et plans de Montsoreau signés Hardion et Salleron, documents iconographiques concernant l'histoire de la «Dame de Monsoreau " (vues de monuments, fac-similés de portraits du temps exécutés par Jean Clouet, conservés au musée de Chantilly), planches de monuments anciens du Maine-et-Loire et de la Mayenne extraites de l'ouvrage du baron de Wismes.

- Archives sonores : a été notamment poursuivie la collecte de témoignages relatifs à la Seconde Guerre mondiale. Sont également entrés par don une importante enquête portant sur les jeunes filles des milieux populaires ruraux en Anjou, ainsi que le témoignage de Philippe Mornet, architecte.

\section{2) Instruments de recherche et publications}

Suite aux classements d'archives aboutis en 2011, on peut signaler les inventaires suivants : 1940);

- 2 Y 2 : maison centrale de Fontevraud, dossiers de prisonniers (1800-

- état des fonds du Conseil général de Maine-et-Loire depuis 1940;

- $2336 \mathrm{~W}$ : Inspection académique, division des examens et concours (1940-2007);

- 1 W-dépôt : haras national du Lion-d'Angers (1800-1993);

- 13 ETP 1 : Ecole de sages-femmes René-Rouchy d'Angers (1839-2007);

- $25 \mathrm{AV}$ : association pour la promotion et l'intégration dans la region d'Angers, témoignages oraux sur le quartier de Monplaisir à Angers et la mémoire des migrations à Trélazé (1994-1997);

- 45 AV : témoignage de Philippe Mornet, architecte (1998-1999);

- $321 \mathrm{~J}$ : association pour la sauvegarde de l'enfance et de l'adolescence de Maine-et-Loire, archives sonores et audiovisuelles (1974-1996).

Archives conservées dans les communes et ayant fait l'objet d'un classement et d'un inventaire par un archiviste contractuel :

Andard, Avrillé, Beaucouzé, Beausse, Bourg-l'Evêque, Brain-surl'Authion, Breil, Challain-la-Potherie, Chalonnes-sous-le-Lude, Chalonnes- 
sur-Loire, Châtelais, Cheffes, Chemellier, Chigné, Corzé, Courléon, Feneu, Fontevraud-l'Abbaye, La Poitevinière, La Possonnière, La Renaudière, Le Longeron, Saint-Barthélémy-d'Anjou, Saint-Crespin-sur-Moine, SaintGeorges-des-Sept-Voies, Saint-Georges-sur-Layon, Saint-Quentin-en-Mauges, Torfou, Varennes-sur-Loire, Villevêque.

\section{Bases de données}

- Bibliothèque

- Journaux et périodiques

- Fonds iconographiques

- Notaires

\section{3) Échos et nouvelles}

\section{Expositions}

Parmi les manifestations que les Archives départementales ont organisées en 2011, on peut citer les expositions Décors et enluminures, Anjou Vélo Vintage, Chevaux et cavaliers à Saumur.

\section{Activités pédagogiques}

Le service éducatif a connu une forte activité, avec près de 3700 élèves accueillis en 2011. Huit établissements scolaires ont par ailleurs participé à la quinzième édition du concours " Montrer l'histoire " organisé par les Archives départementales.

\section{Numérisation}

Le corpus de documents numérisés s'est étoffé en 2011, avec la mise en ligne des registres matricules militaires. Ils viennent s'ajouter aux fonds déjà accessibles via le site internet :

- plans cadastraux napoléoniens

- état civil

- presse : Les Affiches d'Angers (1773-1811) et Le Petit Courrier (18961944)

- plans d'architectes

- cartes postales

- collection iconographique et Dictionnaire de Maine-et-Loire de Célestin Port

- délibérations du conseil général (1799-1982). 


\section{Archives départementales de la Mayenne}

\begin{tabular}{|c|}
\hline Fiche signalétique \\
Archives départementales de la Mayenne \\
6 place des Archives, BP 80744 - 53000 LAVAL \\
Téléphone : 02 43591090 - Télécopie : 0243597771 \\
e-mail : archives@cg53.fr \\
Site internet : www.lamayenne.fr \\
Heures d'ouverture : du lundi au vendredi de $9 \mathrm{~h} 00$ à $17 \mathrm{~h} 00$, samedi (hors \\
vacances scolaires) : de $9 \mathrm{~h} 00$ à $12 \mathrm{~h} 00$ et de $13 \mathrm{~h} 30$ à $16 \mathrm{~h} 00$
\end{tabular}

\section{1) Enrichissement des collections}

\section{Archives publiques}

Plusieurs fonds importants d'archives publiques sont entrés au cours de l'année 2011 aux Archives départementales de la Mayenne.

Dans le domaine des archives hospitalières, l'entrée des archives de l'hôpital spécialisé de la Roche-Gandon à Mayenne est l'évènement le plus marquant de l'année. Un versement de $20 \mathrm{ml}$ de documents de 1900 à 1970 est en effet venu compléter les archives du XIX ${ }^{\mathrm{e}}$ siècle entrées aux Archives départementales en 1994.

Quant aux dépôts des archives des communes, si leur nombre diminuent c'est en raison du taux de dépôt atteint par les Archives de la Mayenne qui conservent désormais plus de $95 \%$ des archives anciennes des communes du département. L'intérêt des fonds déposés lui, ne se dément pas : le fonds très complet de Villaines-la-Juhel (1670-1920), les archives de Charchigné depuis 1624, celles de Bais depuis l'an V, de Thubœuf depuis 1745, ou de Saint-Denis-d'Anjou (an X-1955), sont désormais accessibles aux chercheurs.

Les archives notariales se sont également accrues grâce aux versements des études de Ballots, Cossé-le-Vivien, Craon, Laval et Villaines-la-Juhel (archives des $\mathrm{XIX}^{\mathrm{e}}$ et $\mathrm{XX}^{\mathrm{e}}$ siècles représentant environ 35 mètres linéaires).

Les versements d'archives contemporaines (services de l'État, services du conseil général, établissements publics, etc.) ont été beaucoup moins nombreux que les années précédentes mais on peut noter l'entrée des archives de l'Office public départemental des habitations à loyer modéré (OPDHLM) depuis 1948 (20 ml).

En ce qui concerne les fonds judiciaires, un versement très important des tribunaux d'instance de Laval, Mayenne et Château-Gontier (plus de $40 \mathrm{ml}$ de 1964 à 2006) a été effectué en 2011.

Enfin, trois fonds d'écoles primaires (registres matricules des élèves principalement) ont pu être collectés au cours de l'année : Saint-Loup-duGast, Saint-Denis-d'Anjou, Saint-Thomas-de-Courceriers (XIX'-XX' 


\section{Archives privées}

Le classement du très volumineux fonds de l'architecte Anne Dazelle, donné aux Archives départementales en 2008, a été entrepris. On y trouve notamment les dossiers concernant le secteur sauvegardé de la ville de Château-Gontier.

Une collection privée de cartes géographiques anciennes, exposée au château de Sainte-Suzanne du 8 juillet au 6 novembre 2011, a été numérisée à titre de complément par les Archives départementales. Ce magnifique ensemble s'échelonne de 1525 à 1830 environ.

Les documents personnels d'un ancien requis du service du travail obligatoire (STO), également prêtés par un particulier, ont fait l'objet d'une numérisation de complément. Ce fonds vient compléter la documentation déjà existante sur ce sujet aux Archives départementales.

Enfin, un recueil de documents relatifs à Francis Delaisi (1873-1947), écrivain, journaliste et économiste français né à Bazougers en Mayenne, a rejoint les collections départementales.

\section{Archives iconographiques et audiovisuelles}

Les fonds iconographiques se sont enrichis d'un album de 66 photographies sur la ville de Laval après les bombardements de 1944, et de 350 cartes postales anciennes qui viennent compléter la collection de plus de 10000 cartes conservées aux Archives de la Mayenne.

\section{2) Instruments de recherche et publications}

\section{Instruments de recherche}

Parmi les travaux de classement réalisés en 2011 (environ $200 \mathrm{ml}$ ), on peut noter notamment le classement :

- du très bel ensemble des archives des écoles normales de Laval (écoles des instituteurs, école des institutrices, école mixte),

- du fonds du Parc de stockage des alcools de Longuefuye, établissement public national ayant fermé ses portes le 31 décembre 2010,

- de plus de $80 \mathrm{ml}$ d'archives notariales.

\section{Bases de données}

Pas moins de 882 instruments de recherche ont été publiés dans le moteur de recherche Archinoë et mis à la disposition du public en salle de lecture. Ce corpus d'instruments de recherche concerne les archives publiques contemporaines (postérieures à 1940). 


\section{3) Échos et nouvelles}

Le bâtiment des Archives départementales de la Mayenne, ouvert au public en 1993 (architectes : Dominique Perrault et Alfred Heude), a obtenu en 2011 le label " Patrimoine du Xx $x^{\mathrm{e}}$ siècle ». Il s'agit du deuxième édifice labellisé dans le département; le premier étant le monastère du Carmel à Laval.

Joël Surcouf, directeur des Archives départementales de la Mayenne depuis 1974, a quitté ses fonctions le 2 juin 2011, date de son départ en retraite.

\section{Site internet, expositions et publications}

Les statistiques de fréquentation du site internet sont reparties à la hausse après un léger fléchissement en 2010 et pendant les premiers mois de l'année 2011. Le nombre d'images consultées en 2011 (25475346) dépasse celui de l'année 2010 (24917483).

La mise en ligne de documents d'archives s'est poursuivie avec notamment les 269 monographies communales de 1900, qui participent pour une large part à l'augmentation de la fréquentation du site. La mise en ligne des registres d'ordre des conservations des hypothèques de Laval et de Mayenne, après ceux de Château-Gontier, a l'avantage de permettre aux professionnels (notaires et généalogistes professionnels, notamment) d'effectuer eux-mêmes leurs recherches à distance.

En 2011, les Archives de la Mayenne n'ont réalisé ni exposition ni publication. Elles ont en revanche accueilli l'historien Antoine Prost - invité par le groupe de recherche sur le mouvement social en Mayenne - pour une conférence qui a réuni environ 70 personnes sur le thème L'enseignement des années 1930 à Vichy, ruptures et continuités.

Les journées du patrimoine ont-elles aussi rencontrées un franc succès : 540 visiteurs ont découvert les locaux, les missions des Archives, les archives numérisées ainsi que des documents « remarquables " exposés à cette occasion.

\section{Numérisation}

Parmi les documents dont la restauration et la numérisation ont été réalisées cette année, on retiendra un exemplaire du Dictionnaire historique, topographique et biographique de la Mayenne ayant appartenu personnellement à son auteur, l'abbé Angot, et annoté de sa main. Cet ouvrage, en sept volumes, avait beaucoup souffert des multiples manipulations dont il avait été l'objet; sa numérisation et sa restauration le mettent définitivement à l'abri de toute nouvelle dégradation.

Les autres travaux de numérisation (registres des délibérations des conseils municipaux, registres des hypothèques, archives de la période révolutionnaire) se sont poursuivis.

Enfin, les trois volumes manuscrits des mémoires d'Auguste-Symphorien Alleaume (père du maître-verrier mayennais Auguste Alleaume) ont été également numérisés au cours de l'année. 


\section{Archives départementales de la Sarthe}

\section{Fiche signalétique}

\section{Archives départementales de la Sarthe}

9 rue Christian-Pineau - 72100 LE MANS

Téléphone : 0243547474 - Télécopie : 0243547446

e-mail : archives@sarthes.com

Heures d'ouverture : lundi, mercredi à vendredi de $8 \mathrm{~h} 30$ à $17 \mathrm{~h} 30$; un samedi tous les mois et demi, $8 \mathrm{~h} 30-13 \mathrm{~h} 00$

(fermeture annuelle la $1^{\text {re }}$ semaine de septembre et du 25 décembre au $1^{\text {er }}$ janvier)

\section{1) Enrichissement des collections}

\section{Archives publiques}

Dans le fil des deux années précédentes, marquées par une inflation des versements d'archives publiques (856 mètres linéaires en 2009 et 1100 mètres linéaires en 2010) dans le contexte de la révision générale des politiques publiques et de reprises d'arriérés de versements, les Archives départementales ont accueilli 882 mètres linéaires de documents en 2011.

Parmi ces 67 versements, on relève plus particulièrement les archives des :

- Caisse primaire d'assurance maladie, caisse d'allocations familiales (1921-1995);

- Centre d'accueil pour demandeurs d'asile (1990-2005);

- Agence départementale de développement économique et touristique (1965-2008).

\section{Archives privées}

Le Conseil général a fait l'acquisition en 2011 :

- album de photographies réalisées au prytanée militaire de La Flèche, en 1853-1854, par Jean-Marie Taupenot, professeur de physique et inventeur du procédé d'impression sur papier albuminé au collodion sec. Ce document doit être rapproché de "l'album Napoléon " conservé au prytanée militaire;

- cartons de vitraux à thématique religieuse, rescapés de l'atelier des maîtres verriers Fialeix, établis au Mans autour de 1840 puis à Mayet. Ces cartons feront l'objet d'une opération de nettoyage et de consolidation puis d'une numérisation en vue de leur mise à la disposition du public;

- plan aquarellé de la gare du Mans.

On notera également un dépôt de la Coopérative agricole de la Sarthe (1932-1993) et de la Société de secours mutuels de La Suze-sur-Sarthe (18781930). 


\section{2) Instruments de recherche}

24 instruments de recherche analytiques et 15 inventaires synthétiques ont été rédigés au cours de l'année.

\section{3) Échos et nouvelles}

\section{Expositions}

Une exposition consacrée à la Vallée de la Sarthe artistique et pittoresque, vue au travers du regard d'artistes (peintres, aquarellistes, dessinateurs), conçue sur une idée originale des Archives de l'Orne, a été présentée aux Archives départementales de juillet à octobre 2011.

Les Archives de la Sarthe ont apporté leur contribution à la réalisation d'une exposition consacrée à Deux cents ans de protestantisme en Sarthe, présentée au temple du Mans par l'Église réformée de France, à l'occasion des journées européennes du patrimoine.

\section{Activités pédagogiques}

700 élèves du secondaire et 900 élèves en visites de groupes ont été accueillis par les enseignants du service éducatif et les médiateurs pédagogiques des archives, au cours de l'année scolaire 2010-2011.

Comme en 2010, les Archives départementales ont organisé, à l'initiative de l'Inspection académique et en partenariat avec l'ONAC et les associations d'anciens combattants, une demi-journée de mobilisation du monde enseignant autour du concours national de la Résistance et de la Déportation le 12 octobre 2011.

\section{Fréquentation de la salle de lecture}

Fréquentation de la salle de lecture

1128 lecteurs ont été accueillis en 2011, à l'occasion de 3769 séances de travail (en hausse de 9,7 \% par rapport à 2010), soit 15690 documents communiqués. $47 \%$ des visiteurs ont effectué des recherches généalogiques et 27 \% des recherches à caractère scientifique.

\section{Numérisation}

La numérisation des registres matricules militaires s'est poursuivie jusqu'à la classe 1921 (40170 pages). Leur indexation en vue d'une interrogation nominative en ligne sur le site internet des Archives sera réalisée prochainement. 


\section{Archives municipales d'Angers}

Fiche signalétique
Archives municipales d'Angers
Tervice Archives-Documentation-Photothèque de la ville d'Angers
Hôtel de Ville, boulevard de la Résistance-et-de-la-Déportation,
CS 80011 - 49020 AnGERs cedex 02
Téléphone : 0241054281 - Télécopie : 0241053911
e-mail : gilles.neau@ville-angers.fr (secrétariat)
sylvain.bertoldi@ville-angers.fr (direction)
Site internet : www.angers.fr/archives
Heures d'ouverture : du lundi au vendredi de 14 h 00 à $17 \mathrm{~h} 00$
fermeture annuelle la 1 $1^{\text {re }}$ quinzaine d'août

\section{1) Enrichissement des collections}

\section{Archives publiques}

Les versements d'archives se sont élevés à 102,8 ml en 2011. À noter parmi les versements :

- spectacles de rue des Accroche-Cœurs (1999-2005);

- fonds d'intervention culturelle, subventions aux associations (19972005);

- ZAC Gâte-Argent et Front-de-Maine;

- développement durable, organisation du colloque "Rio+10", tri des ordures ménagères.

\section{Archives privées}

Parmi les fonds ou éléments les plus intéressants :

- comptes rendus critiques des spectacles du théâtre par A. Bruel;

- album humoristique de l'exposition de 1895;

- bâtiments du Welcome (actes de propriété);

- congrès du centenaire des établissements Cointreau;

- correspondance de la famille de Beaumont d'Autichamp;

- correspondance du chimiste Joseph Louis Proust (1792-1806);

- licence délivrée au prêtre Simon Gruget pour exercer paroisse de la Trinité (1783).

\section{Archives iconographiques et audiovisuelles}

18495 nouvelles pièces sont entrées dans les fonds iconographiques : versements du photographe de la Ville, reportages photographiques sur Angers et les commerces angevins effectués par les Archives, dons et achats... Il faut noter en particulier :

- cartes postales 1900-1920 (coll. Raymonde Laiyet); 
- photographies des artistes qui se sont produits à Angers (coll. R. Laiyet);

- aquarelles angevines de Jules et René Rohard.

\section{Bibliothèque}

La bibliothèque d'histoire locale s'enrichit constamment, par dons et achats. Une bibliothèque d'histoire administrative ancienne est également disponible. La collection de périodiques compte 377 titres.

\section{2) Instruments de recherche et publications}

\section{Instruments de recherche}

En plus des classements ouverts en continu pour les séries d'archives qui s'accroissent au jour le jour (versements administratifs contemporains, archives privées, documents iconographiques) et font l'objet d'inventaires, il faut signaler le répertoire numérique détaillé pour le fonds de la Serca-TV 10 (2060 W).

\section{Autres publications}

"La Croix et le tambour : le rôle du corps de ville dans l'organisation de la procession du Sacre ", dans Dies solemnis. Le Grand Sacre d'Angers, Angers, Conseil général de Maine-et-Loire, 2011, p. 84-107.

" Le jeu du papegaut à Angers ", dans Archives d'Anjou, n 15 (2011/2012), p. 5-15.

"Toute la vérité sur ma vie. Souvenirs d'une existence de misère en Anjou, de la Belle Époque aux Années folles ", dans Archives d'Anjou, n 15 (2011/2012), p. 127-138.

"L'hôtel du Roi-de-Pologne ", dans bulletin AVF (Accueil des villes françaises), $\mathrm{n}^{\circ} 3,2012$, p. 26-28.

" Angers en tramway ", dans bulletin AVF (Accueil des villes françaises), $\mathrm{n}^{\circ} 3,2012$, p. 29-31.

Une chronique historique paraît mensuellement dans Vivre à Angers. Une version plus développée est en ligne sur internet à l'adresse indiquée ci-dessus.

\section{3) Échos et nouvelles}

L'année 2011 a été marquée par le réaménagement des locaux des Archives et de la Documentation et leur extension à l'hôtel de ville avec la création de nouveaux magasins. La salle de lecture a été fermée pendant cinq mois et les équipes mobilisées une grande partie de l'année par ce chantier. 


\section{Fréquentation de la salle de lectures - nombres de documents communiqués}

218 lecteurs ont consulté 2735 documents. L'ensemble des communications de documents, comprenant celles effectuées au profit des services de la mairie et les clichés numériques envoyés par le service Photothèque, se monte à 7287 pour 2011.

\section{Site internet}

Le site internet est riche de plus de 1800 pages, sans compter les nombreux documents rattachés au format pdf (67 inventaires). Le dictionnaire historique des rues d'Angers en ligne est en cours de refonte.

\section{Numérisation}

Les opérations de numérisation ont porté principalement sur l'état civil de la première moitié du $\mathrm{xx}^{\mathrm{e}}$ siècle et sur la série Obj (objets concernant Angers, médailles, drapeaux...). La collection particulière $s$ sur les manèges forains construits par les ateliers d'André Chéreau a également fait l'objet d'une numérisation.

\section{Participation aux expositions}

- Saint-Serge-Ney-Chalouère, aux racines historiques du quartier

- Le quartier Deux-Croix-Banchais-Grand-Pigeon

- La Société d'Agriculture, Sciences et Arts d'Angers (1828-1845).

\section{Activités pédagogiques}

Des cours de paléographie sont donnés à l'université et, pour tous les passionnés de recherches, dans le cadre de l'Association des Amis des Archives de l'Anjou (4 A). 


\section{Archives Ville Le Mans et Le Mans Métropole}

\begin{tabular}{|c|}
\hline Fiche signalétique \\
Archives municipales et du Mans Métropole, communauté urbaine \\
102, rue Gambetta - 72039 Le Mans cedex 9 \\
Téléphone : 0243474903 - Télécopie : 0243474368 \\
e-mail : archives@ville-lemans.fr \\
Heures d'ouverture : lundi-vendredi, 8 h $30-12 \mathrm{~h} 00$ et $13 \mathrm{~h} 00-17 \mathrm{~h} 00$ \\
(fermeture des portes 15 minutes avant)
\end{tabular}

\section{1) Enrichissement des collections}

\section{Archives publiques}

Plus de 115 mètres d'archives ont été traités alors que 39 mètres de documents ont été éliminés. Parmi les versements les plus notables, citons :

- Assemblées : conseil municipal, 2008-2009, conseil communautaire (2008-2009);

- Cabinet du Maire : création de la $1^{\text {re }}$ ligne de tramway (1989-2009), manifestation publique locale (2005-2008);

- Direction solidarité urbaine : conseil de quartiers, tranquillité publique, développement social des quartiers (1977-2009);

- Espaces verts : aménagement des aires de jeux et des espaces verts (1956-2002);

- Délégation de services publics : gestion des syndicats mixtes et des technopoles, suivi des transports en commun (1997-2011);

- Jeunesse : action dans les quartiers, équipements socio-culturels (1975-2006);

- Médiathèque : animation culturelle, 1996-2008, rapport d'activité et statistique (1959-1990), revue de presse (1981-2006);

- Population : registre des naissances (1911);

- Services généraux : arrêtés du Maire (2010), arrêtés du Président de Le Mans Métropole (2010);

- Théâtre de l'Espal : programmation culturelle (2009-2011);

- Urbanisme : plan d'occupation des sols et plan local d'urbanisme des communes de la Communauté urbaine du Mans (1984-2007), permis de construire des communes de la Communauté urbaine du Mans (1995-2004), permis de construire de la ville du Mans (2002-2003);

- Voirie : sommier provisoire de la voirie urbaine publique et privée (1937-1941).

\section{2) Instruments de recherche et publications}

15836 notices ont été saisies et indexées sur le logiciel de gestion d'archives Avenio qui fait office d'instruments de recherche, tandis que les répertoires numériques existants pour les séries modernes ont été mis à jour. 
Deux articles ont été publiés dans la Revue mancelle et sarthoise de février 2011, "La Garde nationale " par Sophie Rouyer et " Le premier tramway du Mans » par Nadia Le Gal.

\section{3) Échos et nouvelles}

- Poursuite de la campagne de numérisation : bulletins de salaire 2010 des agents et élus de la Ville et Le Mans Métropole; 22 registres d'état-civil (mariages et décès 1923-1932); 11 registres de délibérations du conseil municipal (1916-1930); état de section cadastrale (1844-1848); 1720 reportages photographiques architecture et opération d'urbanisme (1965-1966);

- Poursuite du dépouillement et de l'indexation au dossier des demandes de permis de construire délivrées entre 1954-1957 et 1999-2004, soient 3214 références;

- Dépouillement et indexation des registres de délibérations du conseil municipal, soient 900 références couvrant la période 1863-1868;

- Participation à cinq émissions de radio locale de Radio France sur des thèmes d'histoire locale.

\section{Archives municipales de Nantes}

\section{Fiche signalétique}

Archives municipales de Nantes

1 rue d'Enfer - 44094 NANTES cedex 01

Téléphone : 0240419585 - Télécopie : 0240473879

e-mail : archives@mairie-nantes.fr

Site internet : $w w w$.archives.nantes.fr

Heures d'ouverture : lundi au vendredi : 8 h $30-12$ h 30 et 13 h $30-18$ h 00

\section{1) Enrichissement des collections}

\section{Archives publiques}

En 2011, les Archives municipales ont reçu les versements suivants :

- service des espaces verts et de l'environnement;

- CCAS Direction action sociale et insertion;

- élections (1988-2010);

- droits des sols et action foncière (1997-2006);

- Angers-Nantes-Opéra (1945-2003);

- office municipal des sports (2000);

- crédit municipal (1813-2000);

- Nantes-Habitat (1939-2007). 


\section{Archives privées}

Plusieurs fonds privés sont entrés dans les collections par don ou achat parmi lesquels :

- 93 Z Société du cinéma L’Apollo et des cinémas nantais : constitution et fonctionnement (1924-1947)

- 97 Z Fonds Pierre Baudrier (peintre, 1884-1964) : photographies, dessins publicitaires, coupures de presse

- 98 Z Fonds Vallerie : photographies et documents sur cité du GrandBlottereau, usine électrique Lamoricière, mi-carême (1925-1935)

- 99 Z Fonds Brunet-Ollive : 503 plaques de verre, photographies de Nantes et familiales entre deux guerres

- 116 Z Fonds Ginisty : documents de Jacques Ginisty, rugbyman joueur et capitaine du SNUC, photographies, coupures de presse, programmes de match (1936-1958)

- 117 Z Fonds Emille Gouyette : documents d'Emile Gouyette « débombeur ", chef d'équipe artificier au sein de la Défense passive : photographies, rapports d'intervention, correspondance (1939-1945).

\section{2) Instruments de recherche et publications}

En 2011, les notices de documents intégrées à la base de données s'élèvent à 14207 soit :

- 270 notices de parchemins de la série AA

- 445 notices de permis de construire de 1927

- 794 notices d'opérations de Nantes-Habitat

-4164 notices de documents contemporains dont les permis de construire de 1997

- 8277 notices de documents figurés, dont 416 plans, 637 affiches et 7224 photographies

- 2938 notices d'articles de presse ou de périodiques dépouillés

Dans le cadre du démarrage de l'opération de restauration des parchemins, 270 documents (lettres patentes, édits ducaux et royaux 1344-1733) de la série AA ont été décrits. Les images seront jointes à ces notices dans la base de données.

\section{Publications}

- tome 2 de la collection Quartiers à vos Mémoires consacré au quartier Contrie-Durantière

- bulletin n ${ }^{\circ} 5$ du Groupe « Mémoire " du quartier Nantes Sud.

\section{3) Échos et nouvelles}

\section{Site internet}

Le site internet s'est modifié et enrichi à travers : 
- mise en ligne de la base de données des inhumations de l'ensemble des treize cimetières nantais de 1867 à 1961;

- publication d'un dossier documentaire sur l'exposition nationale de 1861 ;

- refonte de la page photothèque du site avec mise en ligne d'albums thématiques, création d'une rubrique actualités (acquisition, don, découverte);

- publication dans la base de données du début de la collection des papiers (factures, courriers) à en-tête sous la cote $37 \mathrm{Fi}$;

- cimetières nantais : publication des notices biographiques des victimes du devoir inhumés dans le carré dédié au cimetière de Miséricorde; - guerre 1914-1918 : publication des notices des soldats apparus dans la rubrique Honneur à nos héros du journal Le Phare de la Loire en 1916-1917.

En 2011 le nombre de pages vues s'élève à 7714098 .

\section{Expositions}

Dans le cadre des Journées du patrimoine, les Archives municipales ont présenté une exposition sur l'exposition nationale de 1861, complétée d'un dossier documentaire sur le site Internet.

\section{Histoire et mémoires des quartiers}

- Circuit découverte du quartier Contrie-Durantière le 20 février 2011.

Plus de 300 personnes ont suivi le parcours ponctué d'arrêts sur les lieux emblématiques du quartier avec lecture des témoignages des anciens habitants par 9 étudiants de la classe d'art dramatique du conservatoire.

- Présentation de l'ouvrage Quartiers, à vos mémoire : de la Contrie à la Durantière.

- "Au temps des ponts ", action intergénérationnelle maisons de retraite de la Madeleine et de la Cerisaie de novembre 2010 à juin 2011.

10 jeunes volontaires en service civil au sein de l'Association UniCités ont mené une collecte de témoignages auprès des résidents avec le concours des Archives municipales. Le 25 mai 2011, le film et le livret réalisés ont été présentés dans le jardin de l'EHPAD de la Madeleine.

- Accompagnement du Groupe mémoire du quartier Nantes-Sud avec la réalisation du bulletin $n^{\circ} 5$ sur le Clos-Toreau, bulletin présenté lors de la manifestation Jardi'n Jazz le 28 mai 2011 dans le parc de la Crapaudine.

\section{Actions pédagogiques}

524 élèves ont été accueillis pour des visites et des ateliers dont celui intitulé Le quartier de mon école : hier-aujourd'hui. 


\section{Numérisation}

L'état civil, précédemment arrêté en 1907, a été numérisé jusqu'en 1911 pour les naissances et 1935 pour les mariages et décès.

L'autre importante opération de numérisation a porté sur un ensemble de documents de la Première Guerre mondiale : affiches, diplômes, registres des hôpitaux militaires, bilans de la municipalité, livre d'or des Nantais morts au combat, rapports annuels des directeurs et directrices des écoles publiques nantaises et fichier des réfugiés accueillis à Nantes.

Enfin 36 titres de presse (nationale et locale) de 1848 ont été numérisés et ont fait l'objet d'un traitement OCR.

\section{Restauration}

Plusieurs interventions ont été réalisées, en particulier le début de la restauration des parchemins (lettres patentes, édits ducaux et royaux conservés en série AA).

Un ensemble de 271 grands plans d'alignement et de nivellement du $\mathrm{XIX}^{\mathrm{e}}$ a lui aussi été restauré ainsi que plusieurs registres de presse de la Libération. 


\section{TOURAINE}

\section{Archives départementales d'Indre-et-Loire}

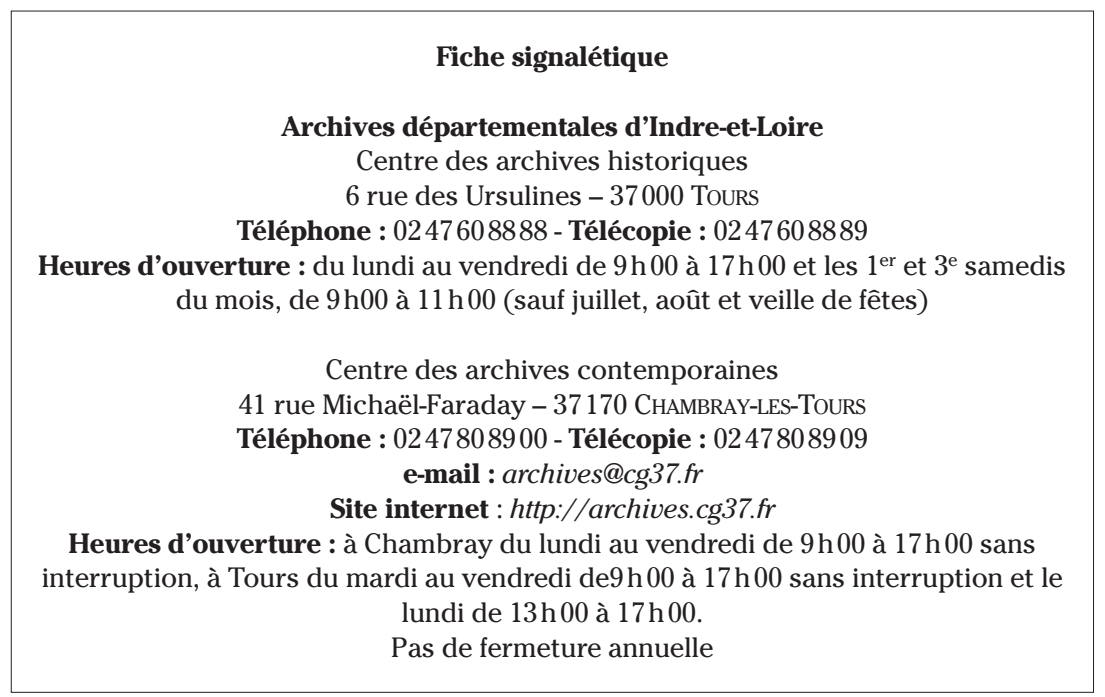

\section{1) Enrichissement des fonds}

\section{Archives publiques}

Pour l'année 2011, il convient de signaler la campagne toujours en cours de collecte de fonds notariaux postérieurs à 1900. Les minutes de la période 1900-1935 font l'objet d'une collecte systématique; les instruments de recherche établis maintiennent une cohérence intellectuelle entre les deux parties du fonds, réparti sur les deux sites des Archives.

Parmi les acquisitions extraordinaires, on citera le fonds de l'imprimerie Mame, qui a fait faillite en 2011. Il représente une dizaine de mètres linéaires mais ne porte que sur une période assez récente.

\section{2) Instruments de recherche et publications}

Le classement de la sous-série 3M (archives des élections, 1800-1940) a été achevé dans l'année et a fait l'objet d'un instrument de recherche dactylographié visé par le Service interministériel des Archives de France.

Deux répertoires de fonds privés d'architectes ayant travaillé en Indreet-Loire ont été finalisés : fonds Lamé-Gasc-Barthélemy-Chalumeau (79 J) et fonds Hess $(185 \mathrm{~J})$. Le classement du volumineux versement $(100 \mathrm{ml})$ consacré aux archives de la délégation départementale du ministère de la Reconstruction et de l'urbanisme (ensuite de la Reconstruction et du 
logement) a été finalisé en janvier 2011. Enfin, le répertoire méthodique du fonds du cadastre rénové, issu du travail réalisé par un conservateurstagiaire de l'Institut national du patrimoine en 2010, a été publié en février.

Concernant les fonds figurés, la base Images de Touraine a connu un accroissement majeur pendant l'été avec la reprise, accompagnée de la mise en ligne, des quelques 7700 notices descriptives de reportages déjà catalogués.

À côté des fonds nouvellement classés, une prestation d'encodage rétrospectif au format XML EAD a été confiée par les Archives à une société privée pour le fonds des archives allemandes collectées à Tours à la fin de la Seconde Guerre mondiale (série non réglementaire ZA); la diffusion de ce répertoire (disponible sur le site Internet des Archives départementales) devrait assurer une exploitation élargie de ce fonds très riche mais peu utilisé par les chercheurs.

\section{3) Échos et nouvelles}

\section{Expositions}

L'exposition sur la ligne de démarcation présentée à la Maison du souvenir de Maillé a rassemblé plus de 4000 visiteurs, tandis que celle consacrée au pont de Chinon a attisé en cette ville la curiosité d'un millier de personnes.

Lors des journées européennes du Patrimoine a été présenté un film sur le thème des Tourangeaux voyageurs, aujourd'hui accessible sur le site Internet des Archives départementales, et une exposition de photographies consacrée aux moyens de transport.

\section{Fréquentation de la salle de lecture}

Le public des Archives d'Indre-et-Loire reste relativement stable par rapport à l'année précédente (1598 inscrits), mais le nombre de séances continue de marquer le pas (6159). L'explication est principalement à chercher du côté de la mise en ligne progressive des registres paroissiaux sur le site Internet des Archives. En effet, une lecture attentive des statistiques montre une chute du nombre de microfilms communiqués (-15\% par rapport à 2010). Cette évolution devrait encore s'accentuer dans les prochaines années, avec la mise en ligne programmé de l'état civil. En revanche, le nombre de documents originaux consultés a augmenté (+ $10 \%)$. La part du public généalogiste dans l'ensemble du lectorat reste pour l'instant constante par rapport à l'année dernière, légèrement au-dessus de la moitié $(54 \%)$. 


\section{Numérisation}

La numérisation des registres paroissiaux d'Indre-et-Loire s'est achevée en 2011, avec la collecte dans les communes puis la numérisation des registres communaux non déposés. L'ensemble ainsi réalisé représente 970000 images numériques. Cette campagne sera suivie en 2012-2013 de la numérisation des registres d'état civil. En interne, la campagne de numérisation porte sur les fonds figurés, notamment sur les nouvelles acquisitions de cartes postales et les reportages du photographe Arsicaud. 